\title{
Influence of Interferon Therapy on Signal-Averaged and Ambulatory Electrocardiograms in Patients With Chronic Active Hepatitis
}

\author{
Shin-ichi HirAmatsu, ${ }^{1}$ MD, Toru MARUYAMA, ${ }^{2}$ MD, Hiroyuki ITO,${ }^{1}$ MD, \\ Shinji ShIMODA, ${ }^{1} \mathrm{MD}$, Yoshikazu KAJI, ${ }^{1} \mathrm{MD}$, and Mine HARADA, ${ }^{1} \mathrm{MD}$
}

\begin{abstract}
SUMMARY
Although interferon (IFN) shows cardiotoxicity and arrhythmogenesis, the influence of IFN on signal-averaged electrocardiography remains to be clarified. The aim of this study was to test a clinical hypothesis that IFN therapy for hepatitis $\mathrm{C}$ virus may induce ventricular late potentials (LPs) and related arrhythmias in patients with chronic active hepatitis. Signal-averaged and ambulatory electrocardiograms were recorded sequentially in patients with chronic active hepatitis $\mathrm{C}(n=22)$ throughout the entire period of IFN therapy. The filtered QRS duration (fQRS) and low amplitude $(<40 \mu \mathrm{V})$ signal duration (LAS40) were significantly increased $(95.5 \pm 8.5$ to $99.6 \pm 9.4 \mathrm{msec}, P<0.0001$, and 32.8 \pm 3.1 to $36.3 \pm 3.0 \mathrm{msec}, P<0.0001$, respectively), whereas the root mean square voltage in the terminal $40 \mathrm{msec}$ of the fQRS (RMS40) was significantly decreased $(25.5 \pm 5.4$ to $22.3 \pm 5.2 \mu \mathrm{V}, P<0.005) 1$ month after starting the IFN therapy. The ventricular LP was negative in all subjects before starting therapy, but became positive in 7 patients after the therapy commenced. There were no differences in clinical baseline characteristics between the LP-positive $(n=7)$ and LP-negative $(n=15)$ groups. Significant increases in mean heart rate, fQRS, and LAS40 were observed after starting the therapy, irrespective of the appearance of the ventricular LP, whereas a decrease in RMS40 was observed only in the LP-positive group. No sustained ventricular arrhythmias were documented in the ambulatory electrocardiography and no cardiac events were encountered in the followup period. Therefore, the results indicate a reversible and subclinical risk of IFN-induced arrhythmogenesis. (Int Heart J 2005; 46: 1033-1040)
\end{abstract}

Key words: Ambulatory monitoring, Interferon, Late potential, Signal-averaged electrocardiography

INTERFERON (IFN) has been widely used in clinical practice as an antiviral, anticancer, and immunomodulatory agent. IFN is particularly effective in the treatment of chronic active hepatitis in patients who are positive for hepatitis $\mathrm{C}$ virus antibody. On the other hand, IFN is known to induce cardiac adverse

From the ${ }^{1}$ Department of Medicine and Biosystemic Science, Kyushu University Graduate School of Medical Sciences, ${ }^{2}$ Institute of Health Science, Kyushu University, Fukuoka, Japan.

Address for correspondence: Toru Maruyama, MD, Institute of Health Science, Kyushu University, Kasuga-Kohen 6-1, Kasuga, Fukuoka 816-8580, Japan.

Received for publication April 22, 2005

Revised and accepted July 14, 2005. 
effects $^{1)}$ such as cardiac dysfunction, ${ }^{2)}$ cardiomyopathy, ${ }^{3)}$ various kinds of arrhythmias, ${ }^{4)}$ and sudden cardiac death, ${ }^{5)}$ although clinical traits which evoke these cardiac events are not documented. In conjunction with these reports, our recent study revealed that human recombinant IFN- $\alpha$ induced conduction slowing and ventricular arrhythmias in isolated and in vivo rat hearts. ${ }^{6}$

Ventricular late potentials (LPs), evaluated by signal-averaged electrocardiography (SAECG), are useful for detecting intraventricular slow or fragmented conduction, which is one of the major arrhythmogenic substrates and otherwise can not be detected noninvasively. ${ }^{7)}$ The aim of this study was, therefore, to test a clinical hypothesis that IFN therapy for hepatitis $\mathrm{C}$ virus may induce ventricular arrhythmogenesis reflected by ventricular LPs and evoke related arrhythmias in patients with chronic active hepatitis.

\section{Methods}

Patients: A total of 22 consecutive patients ( 17 men and 5 women; mean age, 48 \pm 6 years, range, 33 to 59 years) with chronic active hepatitis were enrolled in this study between February 1998 and December 2001. A diagnosis of chronic active hepatitis was made based on the results of biochemical, histological, and serological examinations, including increased antibody and RNA levels for hepatitis C virus in the pretreatment serum. None of the patients had complained of cardiovascular symptoms. The clinical findings, in addition to routine cardiac examinations such as standard ECG, chest X-rays, and transthoracic echocardiography, indicated no structural heart diseases in any of these patients.

Protocols: SAECGs were prospectively recorded with a vector magnitude method (VCM-3000, Fukuda Denshi Inc, Tokyo, Japan) and analyzed in a timedomain manner. Signal averaging was performed using the 3 standard bipolar orthogonal X, Y, and Z leads until a noise level of less than $0.5 \mu \mathrm{V}$ was reached. Two hundred consecutive sinus beats triggered by an $\mathrm{R}$ wave were acquired, digitized, and filtered using a bidirectional digital filter with a bandpass of 40-250 Hz. Data acquisition was performed automatically with a QRS morphology identification correlation coefficient greater than 0.98 . Three parameters related to the ventricular LP were calculated by an automated algorithm, ie, filtered QRS duration (fQRS, msec), root mean square voltage in the terminal $40 \mathrm{msec}$ of the fQRS complex (RMS40, $\mu \mathrm{V})$, and low amplitude $(<40 \mu \mathrm{V})$ signal duration (LAS40, $\mathrm{msec})$. LP in the time-domain analysis was considered to be positive when at least two of the following three conditions met the criteria: fQRS > $120 \mathrm{msec}$, RMS40 $<20 \mu \mathrm{V}$, and LAS40 > 38 msec. $^{8}$ )

Practical SAECG recording was performed with the subjects in a supine position after 10 minutes of bed rest. SAECGs were recorded before, 1 week, and 
1 month after starting the IFN therapy in the morning (10:00 to 11:00 AM), to avoid the IFN-induced febrile period. Two-channel ambulatory ECG (Fukuda Denshi) monitoring was performed immediately after SAECG recording in all subjects. A subcutaneous injection of IFN (human recombinant type $\alpha \mathrm{II}$ or $\alpha \mathrm{IIb}$ ) was administered every day during the 2 week hospitalization period and continued thereafter three times a week in the outpatient clinic for 5 months. In 10 patients SAECGs were also recorded in the outpatient clinic after the cessation of IFN therapy.

Ethics: This prospective study conformed strictly to the Declaration of Helsinki II and the study protocol was approved by the local ethics committee. Written informed consent was obtained from each participant at the time of enrollment in the study.

Statistics: Data are expressed as the mean \pm SD. Statistical analysis was performed using the paired or unpaired Student's $t$-test, as appropriate. Scattered values, such as the frequency of premature ventricular contractions (PVCs), are expressed logarithmically, and $P<0.05$ was considered to be statistically significant.

\section{RESULTS}

In the pretreatment period, the antibody for hepatitis $C$ virus was positive and RNA of this virus was detectable in all subjects. Characteristic lesions of chronic active hepatitis in the biopsied specimens were confirmed in all. All patients responded well to the IFN therapy and no patient was excluded from the study. Fever observed a few days after the initiation of IFN therapy in several subjects was tolerable.

SAECGs were recorded with a mean rejected count of $3 \pm 2$ beats. There were significant increases in fQRS, and LAS40 ( $95.5 \pm 8.5$ to $99.6 \pm 9.4 \mathrm{msec}, P$ $<0.0001$, and $32.8 \pm 3.1$ to $36.3 \pm 3.0 \mathrm{msec}, P<0.0001$, respectively), whereas RMS40 was significantly decreased $(25.5 \pm 5.4$ to $22.3 \pm 5.2 \mu \mathrm{V}, P<0.005) 1$ month after starting IFN therapy in these subjects $(n=22)$. Ventricular LP was negative in all subjects before IFN therapy but was positive in 7 of the 22 patients after starting the therapy. As shown in the Figure, the positive LP was based on the increased LAS40 and decreased RMS40 in these 7 patients. Therefore, the subjects were divided into two groups depending on the appearance of LPs during the therapy. Table I shows there were no statistical differences in the clinical baseline characteristics or results of the noninvasive cardiac examinations between the LP-positive $(n=7)$ and LP-negative $(n=15)$ groups.

Table II presents the sequential changes in mean heart rate, the three indices of ventricular LP, and frequency of PVCs during the course of IFN therapy. The 


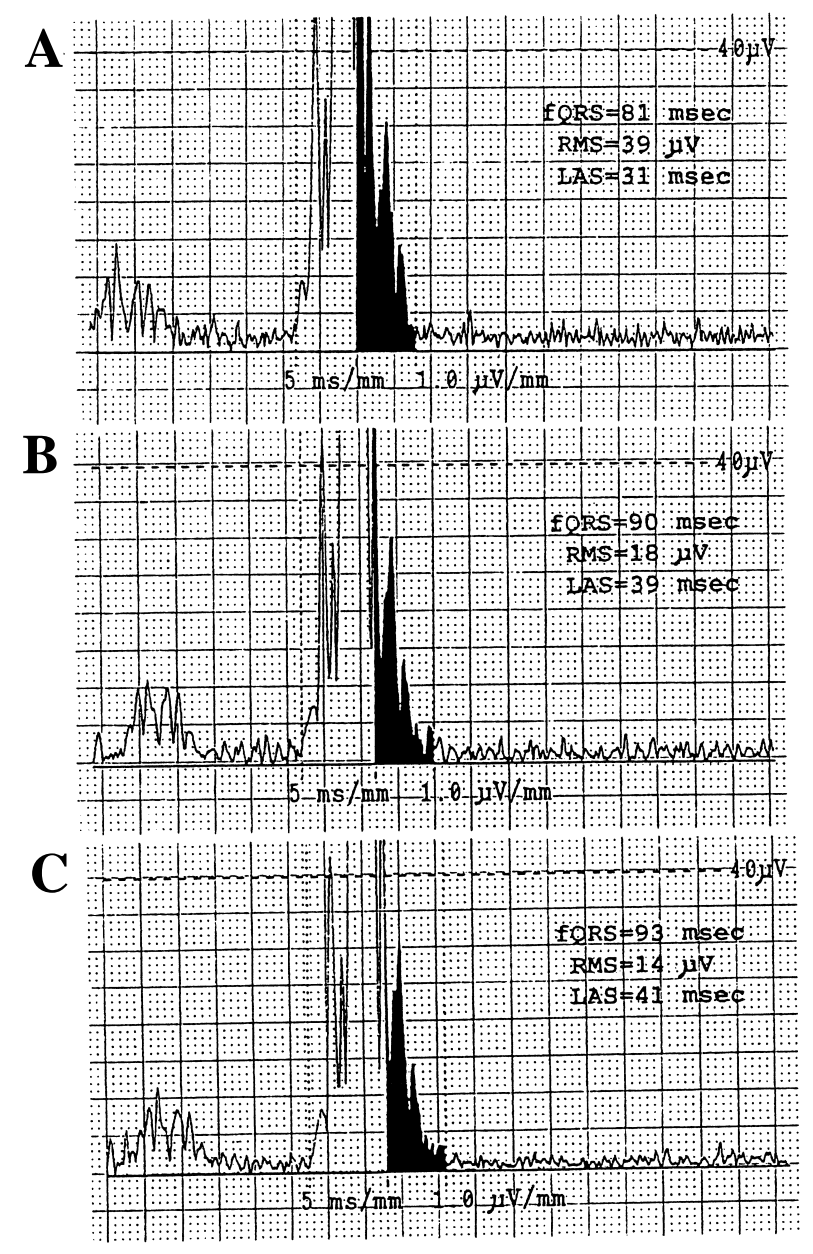

Figure. Signal-averaged electrocardiogram of a 38 -year-old male with chronic active hepatitis before (A), 1 week $(\mathbf{B})$, and 1 month $(\mathbf{C})$ after starting the interferon therapy. The ventricular late potential was negative before and positive after starting the treatment.

mean heart rate in the LP-positive group evaluated by ambulatory ECG monitoring was statistically the same as that in the LP-negative group throughout the entire period of IFN therapy. However, it was significantly increased after starting the treatment in both groups. With respect to the three SAECG indices related to the ventricular LP, the fQRS showed no intergroup differences over the entire treatment period. However, significant increases in the fQRS were observed after starting the therapy, irrespective of the presence or absence of the ventricular LP. The RMS40 exhibited a significant reduction during the therapy in the LP-posi- 
Table I. Clinical Baseline Characteristics of Patients With Positive Versus Negative Ventricular Late Potentials (LPs) Caused by Interferon (IFN) Therapy

\begin{tabular}{lcc}
\hline & $\begin{array}{c}\text { LP-positive group } \\
(n=7)\end{array}$ & $\begin{array}{c}\text { LP-negative group } \\
(n=15)\end{array}$ \\
\hline $\begin{array}{l}\text { Systolic blood pressure }(\mathrm{mmHg}) \\
\text { Diastolic blood pressure }(\mathrm{mmHg})\end{array}$ & $138 \pm 7$ & $133 \pm 5$ \\
Chest X-ray & $83 \pm 4$ & $81 \pm 3$ \\
$\quad$ Cardiothoracic ratio (\%) & $46.7 \pm 4.8$ & $44.9 \pm 4.2$ \\
Standard ECG & $3.7 \pm 0.4$ & $3.5 \pm 0.4$ \\
$\quad \mathrm{~S}_{\mathrm{V} 1}+\mathrm{R}_{\mathrm{V} 5}(\mathrm{mV})$ & $35.3 \pm 2.1$ & $34.8 \pm 1.9$ \\
Echocardiography & $43.0 \pm 1.7$ & $42.6 \pm 1.5$ \\
$\quad$ LAD (mm) & $30.5 \pm 1.4$ & $29.6 \pm 1.1$ \\
$\quad$ LVDd (mm) & $68.7 \pm 4.3$ & $69.1 \pm 4.0$ \\
$\quad$ LVDs (mm) & $10.8 \pm 1.3$ & $10.7 \pm 1.2$ \\
$\quad$ LVEF (\%) & $10.2 \pm 1.2$ & $10.2 \pm 1.2$ \\
$\quad$ IVS (mm) & \\
$\quad$ LVPW (mm) & \\
\hline Data are the mean \pm SD. IVS indicates interventricular septal thickness; \\
LAD, left atrial dimension; LVDd, left ventricular dimension in diastole; \\
LVDs, left ventricular dimension in systole; LVEF, left ventricular ejection \\
fraction; and LVPW, left ventricular posterior wall thickness. No significant \\
differences were noted in the clinical baseline characteristics between the \\
two groups before IFN therapy.
\end{tabular}

Table II. Signal-averaged and Ambulatory Electrocardiograms Over the Entire Period of IFN Therapy in Patients With Chronic Active Hepatitis

\begin{tabular}{lccc}
\hline & Before IFN therapy & One week after starting IFN therapy & One month after starting IFN therapy \\
\hline MHR $(\mathrm{bpm})$ & $72.1 \pm 4.4$ & $77.4 \pm 2.4^{\ddagger}$ & $75.7 \pm 3.5^{\dagger}$ \\
& $71.2 \pm 5.1$ & $76.3 \pm 4.5^{\S}$ & $74.9 \pm 4.4^{\S}$ \\
fQRS $(\mathrm{msec})$ & $93.3 \pm 10.8$ & $96.6 \pm 11.6$ & $98.6 \pm 12.6^{*}$ \\
& $96.5 \pm 7.4$ & $99.3 \pm 8.1^{\ddagger}$ & $100.1 \pm 8.1^{\ddagger}$ \\
RMS40 $(\mu \mathrm{V})$ & $24.7 \pm 5.0$ & $20.3 \pm 3.4^{*} \mathrm{a}$ & $17.9 \pm 1.8^{* \mathrm{~b}}$ \\
& $25.9 \pm 5.8$ & $25.5 \pm 5.8$ & $24.4 \pm 4.9$ \\
LAS40 (msec) & $33.6 \pm 3.4$ & $37.4 \pm 1.6^{*}$ & $39.0 \pm 1.3^{\text {c }}$ \\
& $32.4 \pm 3.0$ & $34.2 \pm 4.0^{*}$ & $35.1 \pm 2.8^{\S}$ \\
log10 [PVCs] & $1.50 \pm 0.37$ & $1.37 \pm 0.56$ & $1.42 \pm 0.45$ \\
& $1.62 \pm 0.38$ & $1.66 \pm 0.34$ & $1.60 \pm 0.45$ \\
Cases showing LP & 0 & 2 & 5 \\
& 0 & 0 & 0
\end{tabular}

Data are the mean \pm SD. The upper data are from the LP-positive group $(n=7)$ and the lower data are from the LP-negative group $(n=15)$. fQRS $=$ filtered QRS duration; LAS40 $=$ low amplitude $(<40 \mu \mathrm{V})$ signal duration; MHR $=$ mean heart rate; PVCs = premature ventricular contractions; and RMS40 = root mean square voltage in terminal $40 \mathrm{msec}$ of fQRS. Frequency of PVCs (beats per day) was expressed logarithmically. ${ }^{*} P<0.05,{ }^{\dagger} P<0.01,{ }^{\ddagger} P<0.005$ and ${ }^{\S} P<$ 0.001 versus pretreatment. ${ }^{\mathrm{a}} P<0.05,{ }^{\mathrm{b}} P<0.005$ and ${ }^{\mathrm{c}} P<0.001$ versus $L P-$ negative group at respective point of time. 
tive group $(P<0.05)$, whereas it remained unchanged in the LP-negative group. Significant prolongation of LAS40 was observed after starting the therapy in both groups, and this prolongation was significantly greater in the LP-positive group than in the LP-negative group $(P<0.001)$. Thus, LP was observed in 2 patients 1 week after and in another 5 patients 1 month after starting the therapy.

No sustained ventricular arrhythmias were documented by ambulatory ECG in either group. The frequency of PVCs (beats per day) showed no individual sequential changes and no intergroup differences (Table II). No cardiac events were encountered in any patients in the mean follow-up period of $3.2 \pm 0.7$ (1.9 to 4.0) years.

SAECGs recorded after cessation of the IFN therapy showed negative ventricular LPs for all subjects $(n=10), 4$ of whom were in the LP-positive group.

\section{DISCUSSION}

This prospective but small-sampled study has demonstrated, for the first time, IFN-induced ventricular LPs in patients with chronic active hepatitis. Chronic hepatitis $\mathrm{C}$ virus infection sometimes causes cardiomyopathy ${ }^{9)}$ which may be reversed by IFN..$^{10,11)}$ However, this type of cardiomyopathy was unlikely in the present patients because routine cardiac examinations conducted at the start of the study indicated no organic heart diseases. Ventricular LPs reflect slow or fragmented ventricular conduction, one of the major arrhythmogenic substrates, predicting an increased risk of ventricular arrhythmia and sudden cardiac death especially in patients with coronary artery disease. Because ambulatory monitoring in this study did not document the appearance of sustained ventricular arrhythmias during the course of the IFN therapy, SAECG findings may predict a subclinical risk of arrhythmogenesis induced by IFN. Moreover, serial SAECGs demonstrated that ventricular LPs that were present during IFN therapy disappeared after completion of the entire treatment. Based on these observations, it is likely that the ventricular LPs observed in this study were due to the reversible electrophysiological effects of IFN per se.

IFN exhibits versatile biological activities, and its subcellular signal transduction mechanisms have been widely investigated. ${ }^{12)}$ IFN indirectly activates protein kinase $\mathrm{C}$ (PKC), which is mediated by cross-talk with the Jak-Stat pathway. ${ }^{13)}$ Recently, this hematopoietic pathway has been reported to be present in cardiomyocytes as a signal transduction system for granulocyte colony-stimulating factor (G-CSF), a newly recognized cardioprotective cytokine. ${ }^{14)}$ PKC modulates various ion channels responsible for myocardial conduction, although this is dependent on the different PKC isoforms. ${ }^{15)}$ For instance, PKC activation inhibits both cardiac $\mathrm{Na}$ and $\mathrm{Ca}$ channels by phosphorylating specific site(s) in certain 
domains of channel subunit(s). ${ }^{16,17)}$ Furthermore, PKC degrades connexin43, a major protein of myocardial gap junctions that plays an important role in cell-tocell coupling and communication. ${ }^{18)}$ Correlation of connexin43 expression with ventricular LPs has been reported recently in patients with nonischemic dilated cardiomyopathy, ${ }^{19)}$ leading to the speculation that PKC-induced degradation of connexin43 will favor the development of ventricular LPs. In the multicellular theoretical fiber based on the Luo-Rudy model, the L-type Ca channel but not the $\mathrm{Na}$ channel contributes to the stable slow conduction under the condition of impaired cell-to-cell coupling. ${ }^{20)}$ Therefore, the dual suppression of $\mathrm{Na}$ and $\mathrm{Ca}$ channels by PKC under the critical coupling condition may accelerate the ventricular slow conduction and may underlie the LPs observed under IFN therapy.

IFN has been reported to cause sympathetic nerve activation, ${ }^{21)}$ which was suspected indirectly in this study due to the increase in mean heart rate observed in ambulatory ECG monitoring after starting the IFN therapy (Table II). Sympathetic activation also modulates the results of SAECG, and hence this possibility should be taken into consideration when interpreting the results of this study. Reportedly, sympathetic activation in addition to parasympathetic withdrawal tends to mask the ventricular LP, ${ }^{22,23)}$ whereas indices of SAECG related to the ventricular LP (ie, fQRS, RMS40, and LAS40) are not usually corrected by the instantaneous heart rate. Therefore, the results of this study may have been offset in part by the altered autonomic nerve activity.

Study limitations: This study has demonstrated clinically IFN-induced ventricular LPs based on the insight obtained in our previous basic study. ${ }^{6}$ The aim of this study included an analysis of the clinical traits leading to an IFN-induced ventricular LP. However, this was not elucidated in the present study since the clinical baseline characteristics demonstrated no significant differences between the LPpositive and LP-negative groups (Table I). The many contributing factors affecting SAECGs should be investigated using multivariate analysis applied to a large number of patients. In other words, a future large-scale study is necessary to clarify the role of ventricular LP in risk stratification of IFN-related cardiac events.

With respect to the ambulatory monitoring, heart rate variability analysis may have clarified a possible role for IFN in the alteration of autonomic nerve activities in more detail. However, heart rate variability over a period of more than 5 months was not analyzed in this study. Because basal physical activity has great influence on autonomic nervous tone, this activity during hospitalization is obviously different from that in the outpatient clinic or during daily life at home. Conclusion: This prospective study showed a reversible and subclinical risk of IFN-induced arrhythmogenesis by combining SAECG with ambulatory monitoring. However, to clarify the role of IFN-induced ventricular LP in the risk stratification of cardiac events during IFN therapy, a large-scale study recruiting more 
patients will be necessary.

\section{REFERENCES}

1. Sonnenblick M, Rosin A. Cardiotoxicity of interferon. A review of 44 cases. Chest 1991; 99: 557-61. (Review)

2. Sartoli M, Andorno S, La Terra G, et al. Assessment of interferon cardiotoxicity with quantitative radionuclide angiocardiography. Eur J Clin Invest 1995; 25: 68-70.

3. Cohen MC, Huberman MS, Nesto RW. Recombinant alpha-2 interferon-related cardiomyopathy. Am J Med 1988; 85: 549-51.

4. Martino S, Ratanatharathorn V, Karanes C, Samal BA, Sohn YH, Rudnick SA. Reversible arrhythmias observed in patients treated with recombinant alpha 2 interferon. J Cancer Res Clin Oncol 1987; 113: 376-8.

5. Dickson D. Deaths halt interferon trials in France. Science 1982; 218: 772.

6. Odashiro K, Hiramatsu S, Yanagi N, Arita T, Maruyama T, Kaji Y, Harada M. Arrhythmogenic and inotropic effects of interferon investigated in perfused and in vivo rat hearts: influences of cardiac hypertrophy and isoproterenol. Circulation J 2002; 66: 1161-7.

7. El-Sherif N, Gomes JA, Restivo M, Merha R. Late potentials and arrhythmogenesis. Pacing Clin Electrophysiol 1985; 8: 440-62.

8. Gomes JA, Winters SL, Stewart D, Targonski A, Barreca P. Optimal bandpass filters for time-domain analysis of the signal-averaged electrocardiogram. Am J Cardiol 1987; 60: 1290-8.

9. Matsumori A, Matoba Y, Sasayama S. Dilated cardiomyopathy associated with hepatitis C virus infection. Circulation 1995; 92: 2519-25.

10. Sato Y, Takatsu Y, Yamada T, et al. Interferon treatment for dilated cardiomyopathy and striated myopathy associated with hepatitis $\mathrm{C}$ virus infection based on serial measurements of serum concentrations of cardiac troponin T. Jpn Circ J 2000; 64: 321-4.

11. Kuwata A, Ohashi M, Sugiyama M, Ueda R, Dohi Y. A case of reversible dilated cardiomyopathy after $\alpha$ interferon therapy in a patient with renal cell carcinoma. Am J Med Sci 2002; 324: 331-4.

12. Lerner Research Institute: Interferon stimulated gene database arranged into functional categories: http:// www.lerner.ccf.org/labs/williams/xchip-html.cgi. Accessed October 10, 2005.

13. Uddin S, Sassano A, Deb DK, et al. Protein kinase C- $\delta(\mathrm{PKC}-\delta)$ is activated by type I interferons and mediates phosphorylation of Stat1 on serine 727. J Biol Chem 2002; 277: 14408-16.

14. Harada M, Qin Y, Takano H, et al. G-CSF prevents cardiac remodeling after myocardial infarction by activating the Jak-Stat pathway in cardiomyocytes. Na Med 2005; 11: 305-11.

15. Marx S. Ion channel macromolecular complexes in the heart. J Mol Cell Cardiol 2003; 35: 37-44. (Review)

16. Qu Y, Rogers J, Tanada T, Scheuer T, Catterall WA. Modulation of cardiac $\mathrm{Na}^{+}$channels expressed in a mammalian cell line and in ventricular myocytes by protein kinase C. Proc Natl Acad Sci USA 1994; 91: 3289-93.

17. McHugh D, Sharp EM, Scheuer T, Catterall WA. Inhibition of cardiac L-type calcium channels by protein kinase C phosphorylation of two sites in the N-terminal domain. Proc Natl Acad Sci USA 2000; 97: 12334-8.

18. Saez JC, Nairn AC, Czernik AJ, Fishman GI, Spray DC, Hertzberg EL. Phosphorylation of connexin 43 and the regulation of neonatal rat cardiac myocyte gap junctions. J Mol Cell Cardiol 1997; 29: 2131-45.

19. Kitamura H, Yoshida A, Ohnishi Y, et al. Correlation of connexin43 expression and late ventricular potentials in nonischemic dilated cardiomyopathy. Circ J 2003; 67: 1017-21.

20. Shaw RM, Rudy Y. Ionic mechanisms of propagation in cardiac tissue. Roles of the sodium and L-type calcium currents during reduced excitability and decreased gap junction coupling. Circ Res 1997; 81: 727-41.

21. Yamamoto T, Tsuchihashi T, Hayashi J, Kashiwagi S. Effect of interferon therapy on heart rate and sympathetic nervous system. Fukuoka Igaku Zasshi 1998; 89: 207-15.

22. Goldberger JJ, Ahmed MW, Parker MA, Kadish AH. Assessment of effects of autonomic stimulation and blockade on the signal-averaged electrocardiogram. Circulation 1994; 89: 1656-64.

23. Nakagawa M, Iwao T, Abe H, et al. Influence of autonomic tone on the filtered QRS duration from signal averaged electrocardiograms in healthy volunteers. J Electrocardiol 2000; 33: 17-22. 